Gastroenterologe 2015 - 10:171-173

DOI 10.1007/s11377-015-1001-0

Online publiziert: 21. April 2015

c) Springer-Verlag Berlin Heidelberg 2015

M. Fried ${ }^{1}$ J.F. Riemann ${ }^{2}$

${ }^{1}$ Klinik für Gastroenterologie und Hepatologie, Universitätsspital Zürich

2 em. Direktor der Med. Klinik C am Klinikum Ludwigshafen, c/o Stiftung LebensBlicke

\title{
Diagnostische und therapeutische Herausforderungen proktologischer Erkrankungen
}

Proktologische Erkrankungen gehören zu den alltäglichen und häufig schwierig abzugrenzenden und zu behandelnden Erkrankungen, die eine Vielzahl von medizinischen Disziplinen beschäftigen. In den letzten Jahren sind beachtliche Fortschritte in Diagnostik und Therapie dieser Erkrankungen erzielt worden. Ziel dieses Hefts ist es, den auf diesem Gebiet tätigen Ärzten - den Chirurgen, Internisten, Dermatologen und Gastroenterologen - einen Überblick über die neuesten Entwicklungen mit dem Fokus auf die klinisch wichtigsten Krankheitsbilder, die sowohl diagnostisch als auch therapeutisch anspruchsvoll sein können, zu vermitteln.

Am Anfang steht, wie immer, die ausführliche Anamnese. Sie ist gerade bei proktologischen Erkrankungen unverzichtbar. Die Differenzialdiagnose der häufigen Proktitiden kann schwierig sein, insbesondere steht die Abgrenzung eines Morbus Crohn von einer venerischen Erkrankung im Vordergrund. Dabei nimmt die Häufigkeit von Geschlechtserkrankungen und chronisch-entzündlichen Darmerkrankungen ständig zu und kann den behandelnden Arzt mit Problemen in der Erkennung und Therapie konfrontieren. So mussten aufgrund der zunehmenden Resistenzbildung gegenüber den früher klassischen Antibiotika in den letzten Jahren diverse Antibiotikarichtlinien bei proktologischen Geschlechtserkrankungen, wie z. B. bei der Gonorrhö, angepasst werden.

Die anale Inkontinenz oder Stuhlinkontinenz gehört zu den häufigen Krankheitsbildern, insbesondere bei älteren $\mathrm{Pa}$ tienten, die eine besondere therapeutische
Herausforderung darstellen. Nach einer umfassenden Diagnostik sollte interdisziplinär mit Einbezug von Chirurgen, Gynäkologen, Gastroenterologen und Urologen nach einer für den individuellen Patienten optimalen Therapie gesucht werden.

》) Die anale Inkontinenz sollte möglichst konservativ behandelt werden

Möglichst sollten dabei konservative Verfahren, wie Beckenbodentraining, Biofeedbackverfahren etc., eingesetzt werden, bevor eine chirurgische Intervention, wie eine STARR-Operation oder eine Schrittmacherimplantation, diskutiert wird.

Die Behandlung der Analfisteln, die zu den häufigen Krankheitsbildern in einer proktologischen Praxis gehören, kann große Anforderungen an die behandelnden Ärzte stellen. Wenn konservative Verfahren zur Heilung von Analfisteln nicht zur Abheilung führen, können operative Methoden eingesetzt werden. Dabei hat das Arsenal chirurgischer Therapieoptionen zur Behandlung von Analfisteln eine große Erweiterung erfahren. Von der Fistulektomie bis zur Stammzellinjektion muss der erfahrene proktologische Chirurg das individuell am besten geeignete Verfahren vorhalten und auswählen, wobei die lokale Expertise eine wichtige Rolle spielt.

Nicht zuletzt gehören die Hämorrhoidalleiden zu den am häufigsten diagnostizierten proktologischen Erkrankungen. Eine Vielzahl von nichtoperativen und operativen Therapieverfahren wurde ent- 
wickelt, was darauf hinweist, dass die Suche nach der idealen Behandlungsmethode bis heute nicht abgeschlossen ist. Bei den ambulanten ohne Sedation angewandten Verfahren hat sich die Gummibandligatur durchgesetzt, bei den operativen Verfahren zählt die Stapler-Mukosektomie nach Longo zu den Standardtechniken. Bei isolierten Hämorrhoiden können auch neuere Methoden, wie die Resektion mit LigaSure ${ }^{\circledast}$, eingesetzt werden. Hämorroidenoperationen können auch zu Langzeitproblemen führen; so sind Analstenosen meist durch Hämorroidektomien verursacht. Sie werden in frühen Stadien durch diätetische und abführende Maßnahmen, in höheren Stadien durch eine mechanische Dilatation und falls erforderlich chirurgisch, z. B. durch eine Anoplastik, behandelt.

Das vorliegende Themenheft eröffnet dem proktologisch tätigen Arzt einen Überblick über die Diagnostik und Therapie der häufigsten und zugleich diagnostisch und therapeutisch anspruchsvollsten Krankheitsbilder. Diese Serie von Artikeln soll dem Kliniker einen für seine tägliche Praxis nützlichen Überblick über dieses wichtige Gebiet geben und eine Hilfestellung zur Behandlung der wichtigsten proktologischen Erkrankungen sein. Den Autoren sei es verdankt, diesen Überblick in didaktisch ausgezeichneten konzisen Artikeln vermittelt zu haben.

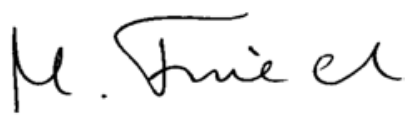

Prof. Dr. M. Fried

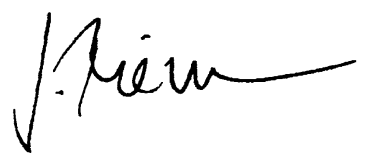

Prof. Dr. J.F. Riemann
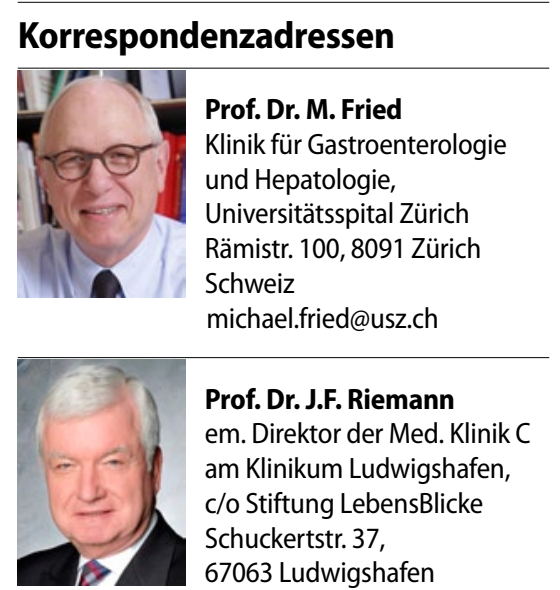

Prof. Dr. J.F. Riemann em. Direktor der Med. Klinik C am Klinikum Ludwigshafen, c/o Stiftung LebensBlicke

Schuckertstr. 37,

67063 Ludwigshafen riemannj@garps.de

Interessenkonflikt. . M. Fried und J.F. Riemann geben an, dass kein Interessenkonflikt besteht.

\section{Krebsfrüherkennung}

Mit dem „Gesetz zur Weiterentwicklung der Krebsfrüherkennung und zur Qualitätssicherung durch klinische Krebsregister" erfolgte in Deutschland eine politische Neuorientierung der Krebsfrüherkennung entsprechend den Empfehlungen der EU und der WHO.

Nichtsdestotrotz ist die Krebsfrüherkennung noch immer ein kontrovers diskutiertes Feld. Das Schwerpunktheft „Krebsfrüherkennung" (Ausgabe 3/2014)

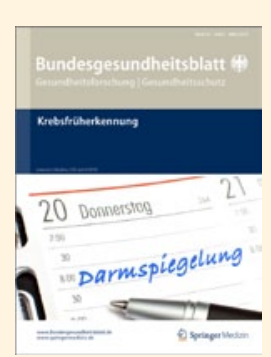
der Zeitschrift Bundesgesundheitsblatt - Gesundheitsforschung - Gesundheitsschutz gibt einen Überblick über den aktuellen Stand, den Nutzen und Schaden

der Früherkennung sowie ethische Wertabschätzungen und methodische Herausforderungen:

- Früherkennung des Zervixkarzinoms in Deutschland

- Krebsfrüherkennung aus Sicht der Public-Health-Ethik

- Prävention von Hautkrebs

- Printmedien- und Internetangebote mit Informationen zur Darmkrebsfrüherkennung und ihre kriteriengestützte Bewertung aus Expertensicht

Bestellen Sie diese Ausgabe zum Preis von 16,- EUR zzgl. Versandkosten bei Springer Customer Service Center Kundenservice Zeitschriften Haberstr. 7

69126 Heidelberg

Tel.: +49 6221-345-4303

Fax: +49 6221-345-4229

E-Mail: leserservice@springer.com

Suchen Sie noch mehr zum Thema? Mit e.Med, dem Online-Paket von Springer Medizin, können Sie schnell und komfortabel in über 600 medizinischen Fachzeitschriften recherchieren. Weitere Infos unter springermedizin.de/eMed. 
Hier steht eine Anzeige.

黛 Springer 